\title{
MARVELOUS BABY: AN OUTCOME OF MULTIDISCIPLINARY APPROACH TO TRAUMATIC BRAIN INJURY IN PREGNANCY DUE TO ROAD TRAFFIC ACCIDENT
} (CASE REPORT)

\author{
By \\ Taha Mohammad Rashad Amer, Yasmine Harby Sadek and Abd El- \\ Hammeed Ezzat Gaber \\ Department of Obstetrics and Gynecology, Sharm International Hospital, Egypt \\ E-mail: tahaamer3000@gmail.com
}

\begin{abstract}
Background: Several literatures supported that trauma complicates 6-7\% of all pregnancies. Despite the rapid progressiveness of traumatic brain injury (TBI) worldwide, there is paucity in literatures regarding the exact incidence of TBI in pregnancy. Management poses a great challenge; the anatomical and physiologic maternal changes due to pregnancy, added to the consideration of existence of two lives till the end.

Objective: To increase the awareness about the management of pregnancy in cases presented with traumatic brain injury during pregnancy.

Case report: We described the management and follow up of a case of 30 years old multiparous female who presented in Sharm International Hospital, on 25-8-2019, at 18weeks gestation, post Road Traffic Accident (RTA) with Glasgow Coma Scale (GCS); 9T (eye opening, localize pain and endotracheal intubated).She was referred from nearby hospital (El Toor General Hospital) with endotracheal tube fixed since about 3 weeks, for tracheostomy and management. The patient was admitted to ICU with file No: 3384, on 25-82019. The patient was very carefully managed in the intensive care unit (I.C.U.) in Sharm International Hospital up to successful vaginal delivery of a live female weighed 1500 grams, at $+/-30$ weeks gestation. As the mother was severely ill and the fetus was extremely premature, we preferred the vaginal mode of delivery which we supposed to be less risky for the mother. The Marvelous baby was handled by pediatrtion team and neonatology doctor. After 3 weeks the baby went home with good crying and on bottle feeding. Unfortunately the mother died 10 days after labor.
\end{abstract}

Key words: Road Traffic Accident (RTA), Traumatic Brain Injury (TBI), Glasgow Coma Scale (GCS), Intensive Care Unit (ICU), pregnancy, fetus.

\section{INTRODUCTION}

Trauma occurs in approximately $7 \%$ of all pregnancies and is the leading cause of non-obstetrical maternal death $(>20 \%)$. The majority of trauma in pregnancy is blunt trauma from motor vehicle accidents, domestic violence and falls. Other reported mechanisms including: penetrating trauma, toxic exposures, burns and self-inflected injuries (Kisilevsky and Flexman, 2018).Traumatic brain injuries (TBI) and hemorrhagic shock are responsible for most maternal fatalities and even minor trauma can be associated with poor fetal outcomes. Indeed, trauma is associated 
with multiple increased risks to the fetus including: abortion, uterine rupture, placental abruption, caesarean section and preterm delivery. In the trauma setting, only $11 \%$ of fetal mortality is attributable to maternal mortality. Early assessment of fetal well-being is paramount. It was proposed that pregnant women, who have significantly elevated levels of estrogen and progesterone, might benefit of the steroid hormones and the hypercoagulability state. But it was found that pregnant patient with moderate to severe TBI showed no statistically significant difference in mortality compared with their non-pregnant counterparts (Berry et. al. 2011).

Objective: To increase the awareness about the management of pregnancy in cases presented with traumatic brain injury during pregnancy.

\section{CASE REPORT}

On 25-8-2019, a 30 year old, multiparous woman, was admitted to Intensive Care Unit( I.C.U.) in Sharm International Hospital, with file No: 3384, referred from nearby hospital (El Toor General Hospital) for tracheostomy and further management. The patient was presented in El Toor General Hospital after Road Traffic Accident (RTA) with Glasgow Coma Scale ( GCS) 9T (eye opening, localise pain and endotracheal intubated),ultrasound examination confirmed the presence of living intrauterine pregnancy with gestational age +/- 18 weeks +/- 4 days. The pregnancy progressed normally as the maternal management continued. Frequent ultrasound examinations revealed neither evidence of major organ trauma in the mother nor free pelvi-abdominal fluid collection. CT- scans of the brain of the mother revealed the presence of brain edema, subarachnoid hemorrhage, multiple brain hemorrhagic contusions and areas of ischemia in the brain. During management in I.C.U., the patient developed status epilepticus; she was eye opening to pain with flexion and spastic all limbs (2/6 epilepsy). She continued on anti-epileptic drugs. Spasticity improved in right side of the body and lower limbs. The patient was feeded by oro-gastric tube and partial parenteral nutrition (PPN). During management she also developed ventilation-associated pneumonia, culture and sensitivity was done and she received the appropriate antibiotic. The patient had silicon urinary bladder catheter fixed and she developed cystitis and properly managed. Also, she developed bed sores grade 2 and properly managed and healed .She was on prophylactic anticoagulant; low molecular weight heparin (Clexan injection $40 \mathrm{mg}$ S.C. daily) and fortunately no deep vein thrombosis developed. As for the cardio-vascular system, she was stable and did not develop shock at any time during management. The patient was exposed during management to CT-brain several times (about 8 times) and X-ray chest every 2 weeks (about 8 times) with use of Lead apron over the abdomen .At about 30 weeks gestation, to induce fetal lung maturity for possibility of preterm labor, the patient received $24 \mathrm{mg}$ Dexamethasone i.v. in two divided doses within 24 hours. Two days later, she developed regular uterine contractions and started to progress as preterm labour spontaneously. As the mother was severely ill and unconscious and the baby was extremely premature; we preferred 
the vaginal mode of delivery which we think to be less risky to the mother. The cervix was unripe; we advised to give Pethedine ampoule $100 \mathrm{mg}$ i.m. and two ampoules Prempran and 1.5 Liters Ringer solutions for hydration. The baby was monitored during the progress of labor for possible occurrence of fetal distress. The labor progressed good and no fetal distress; so we arranged for vaginal delivery. After about 8 hours the patient delivered vaginally an alive female, 1.5 kg. , active and handled by the pediatrtion team. In the Neonate Centre; the baby received 2 ampoules Sevanta immediately (surfactant) (4 mg. / kg.), umbilical cord catheter was fixed. The baby was put on ventilator for 4 days and starts weaning and kept on sepap (nasal oxygen) later on. The baby was fed by oro-gastric tube. The baby progressed well and went home after 3 weeks with good crying and bottle feeding.

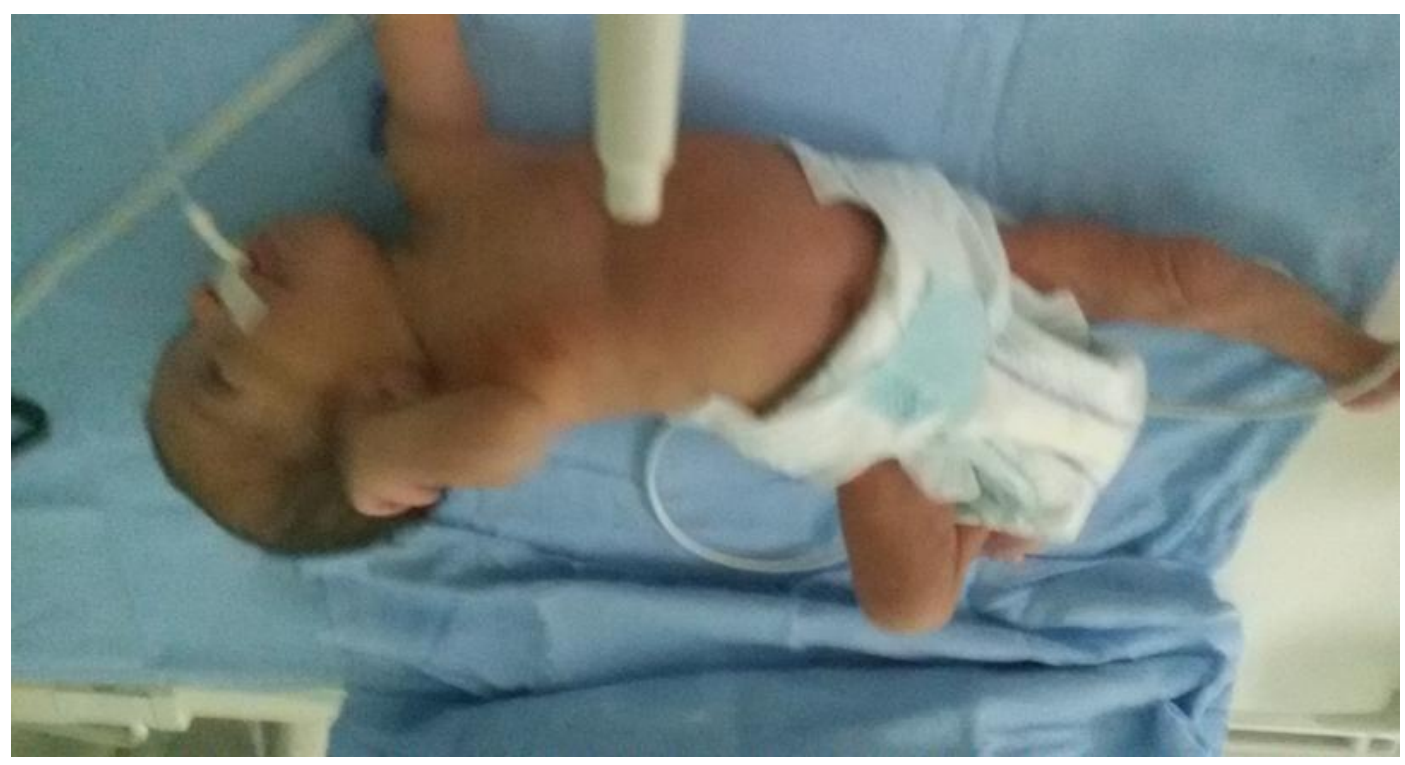




\section{Marvelous Baby \\ DISCUSSION \\ (Desjardins, 2018): Cardiovascular}

TBI warranting neurosurgical intervention in the pregnant population is a rarity. It was reported that a case of sever TBI with extensive hemorrhagic contusions, diffuse brain swelling and multiple skull and facial fractures, progressed good after decompressive craniotomy and managed in the intensive care unit. After near 4 months, at 35 weeks of gestation, the patient had exposed to emergency cesarean section after spontaneous onset of labour. The baby had no detectable abnormality and was clinically well (Nevlle et al., 2012). As for our case, we planned for vaginal delivery with monitoring of the fetal condition to avoid surgical intervention as the patient was severely ill, not fully conscious and under anticoagulant. The patient was multiparous with patulous vagina and the fetus was extremely premature. The labor process progressed well and the miracle baby tolerated the uterine contractions and did not develop any distress during labor. Important system: Rise in cardiac output by $40 \%$ at term pregnancy. Supine position may decrease the cardiac output through reduced venous return. Respiratory system: reduced tidal volume reduces functional residual capacity, persistent compensated respiratory alkalosis and reduced blood buffer capacity. Altered response to inhalation anesthesia is suspected. Hematologic system: reduced hematocrit value and elevated leucocyte count. Hyper coaguability; with elevated risk of thrombosis and emboli. Gastrointestinal tract: Hypomobelity with increased risk of aspiration of gastric fluid. Compartmentalization of abdominal contents; elevating the risk of upper abdominal penetrating injury. Uterus and placenta: $20-30 \%$ shunts; leading to rapid blood loss. Marked increase in uterine size leading to abdominal organs displacement and supine hypotension. There is placental high flow and low resistance index. Management considerations in intracranial

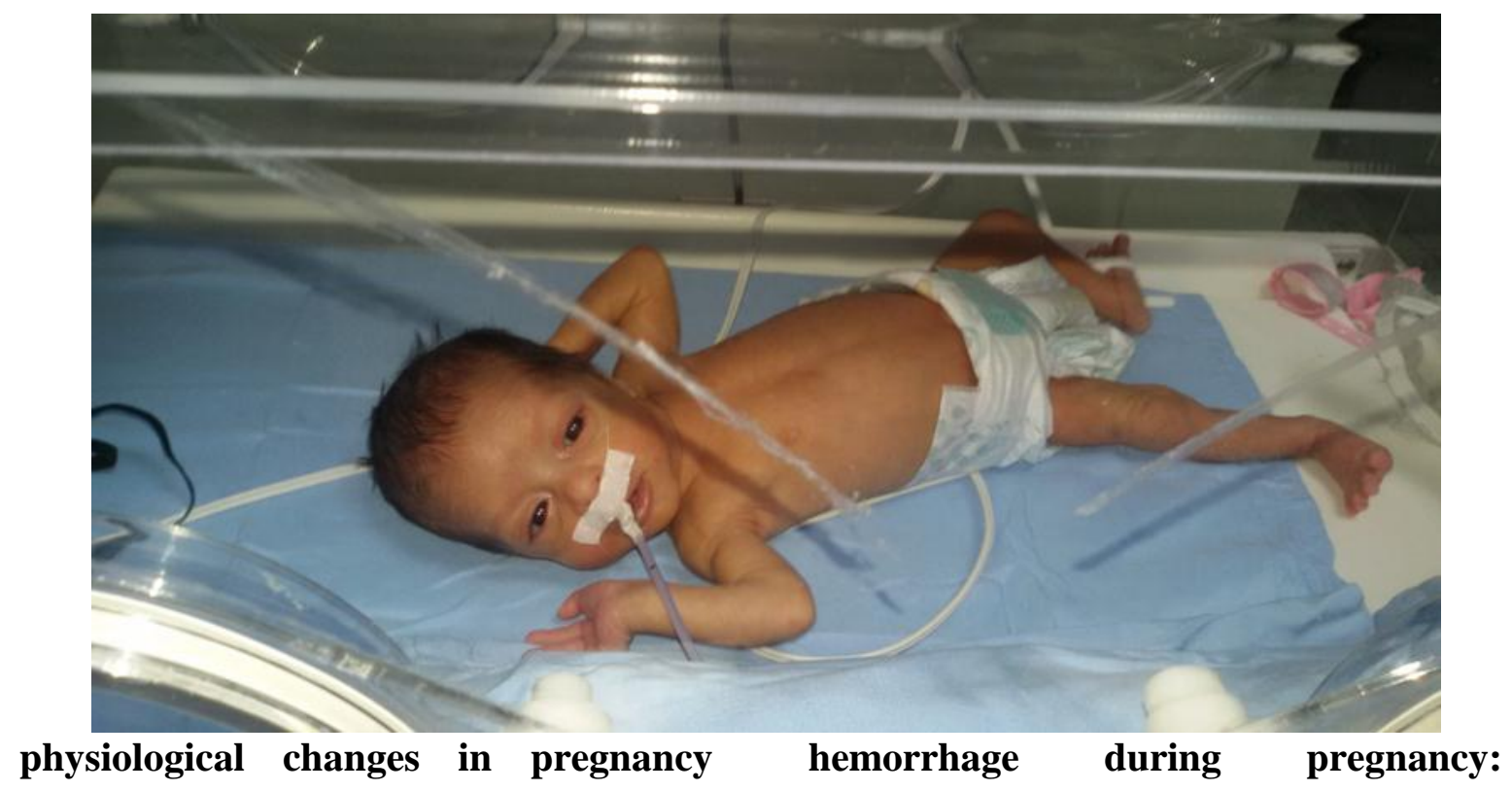


Imaging: diagnostic modality deemed necessary for maternal evaluation should not be withheld on the basis of its potential hazard to the fetus. In general, $x$ ray beams aimed more than $10 \mathrm{~cm}$. Away from the fetus are not dangerous. Studies showed that exposure of the fetus to less than 5-10 rads causes no significant increase in the risk of congenital malformations, intrauterine growth restriction or miscarriage. When performing radiographic evaluation of the mother, fetal irradiation should minimized by shielding the abdomen with a Lead apron. A head CT-scan is always indicated in all head-injured patients. An axial CT-scan without contrast rapidly defines intracranial lesions. Reports from previous studies indicated that radiation dose from A-P chest $\mathrm{X}$-ray is in general below $0.005 \mathrm{rad}$, a pelvic film is below $0.4 \mathrm{rad}$, and CT-scan of the head $(1 \mathrm{~cm}$. slices) is 0.05 rad. Maternal hydration should be emphasized before and after angiography (Desjardins, 2018). Antiepileptics: The general consensus for using antiepileptic drugs is for monotherapy with the lowest possible dose. Concomitant use of folate is strongly recommended. Women with a history of seizures are more prone to seizures during pregnancy, but $>90 \%$ of them has a favorable outcome (Sima and Page, 2016). Anticoagulants: As pregnancy is hyper coagulant state; the use of anticoagulant during management of B.T.I. is recommended in the prophylactic dose. Heparin is a large molecular weight compound and does not cross the placenta. In contrast, coumarine derivatives cross the placenta and have significant teratogenic effects. Warfarine embryopathy syndrome reported to occur in $15-25 \%$ of children born to women exposed to Warfarine in the first trimester (Luesley and Baker, 2010). Glasgow Coma Scale (GCS) according Stratton, 2018: It is a neurological scale that aims to give a reliable and objective way of recording the state of consciousness of a person for initial as well as subsequent assessment. GCS is used in hospitals in monitoring patients in intensive care units (ICU). The scale is composed of three tests: eye opening, verbal response and motor response. The three items separately as well as their sum are considered. The lowest possible GCS is 3 (grade 1 in each element.) denoting coma or death, while the highest GCS is 15 (fully awake person). Rapid assessment and evaluation of pregnant patients with trauma: (Desjardins, 2018 and Setapathy et al, 2014).

\section{(A B C D E F format):}

A. Airway: consider placing laryngeal mask, airway or endotracheal tube if necessary.

B. Breathing: supplemental oxygen in most cases.

C. Circulation: Assess hemodynamic stability, establish two large bore intravenous lines and replace blood loss with isotonic crystalloid solution.

D. Disability: assess an injury severity score and GCS.

E. Expose the patient: remove all clothing and inspect the entire body.

F. Fetal basic assessment: measure fundal height, fetal heart monitoring and gestational age determination.

CONCLUSION and RECOMMENDATIONS 
Over the years, the maternal mortality depending on the obstetric causes has reduced, whereas there is a relative increase in maternal mortality and morbidity due to non-obstetric causes. Trauma during pregnancy, including head injury, is one of the leading causes of incidental maternal death and morbidity and complicating 6-7 \% of all pregnancies. Trauma during pregnancy predisposes two lives at risk (mother and fetus). It creates unique diagnostic and therapeutic challenges because of physiological, hormonal, hemodynamic and anatomical changes associated with pregnancy. Management of TBI with pregnancy always needs multidisciplinary approach to optimize the outcomes for both mother and fetus. If managed conservatively, there should be rational and judicious use of medications with very minimal fetal risks. Surgical management is warranted when its benefits overweight the risks. Proper seat belt use is the most significant modifiable factor in decreasing maternal and fetal injuries and mortality after motor vehicle crashes.

\section{ACKNOWLEDGMENTS}

We would like to express my great thanks to ICU team; who did their best efforts to optimize the outcome for the mother. Also, a lot of thanks for Neonatal team; who did their best with that miracle baby, who went home after 3 weeks with normal breathing and on bottle feeding. Deep thanks for the nursing staffs of both ICU and Neonatology Departments.

\section{REFERENCES}

1. Berry C, Ley E.J. and Mirocha (2011): Do pregnant women have improved outcome after traumatic brain injury? A.J.S., 201 (4): 387-398.

2. Desjardins G. (2018): Initial management of a pregnant woman with trauma, Journal of Obstetric Anaesthesia and Critical Care, 8 (2):66-72.

3. Kisilevsky A. and Flexman A.M. (2018): Pregnancy and Neurotrauma, chapter in book: Essentials of Anesthesia for neurotrauma: $387-398$.

4. Luesley D.M. and Baker P.N. (2010): Obstetrics and Gynecology An evidencebased text for MRCOG, second edition, Edward Arnold publisher: $147 \& 188$ - 195 .

5. Neville G., kaliaperumal C. and Kaar G. (2012): Miracle baby: an outcome of multidisciplinary approach to neurotrauma in pregnancy. BMJ, volume 2012 case report, bcr- 2012-006477.

6. Setapathy M.C., Mishra S.S., Das S. et al (2014): The Indian Journal of Neurotrauma: $11(1): 45-48$.

7. Sima I.P. and Page B.P. (2016): Management of epilepsy during pregnancy: an update, Neurological Disorders, 9 (2): $118-129$

8. Stratton S.J. (2018): Glasgow Coma Scale Score in trauma Triage: A Measurement without Meaning. Ann Emerg Med., 72 (3): $270-271$. 


\section{الطقلة المدهشة: ثمرة التعاون والرعاية متعددة الأطر اف لسيدة

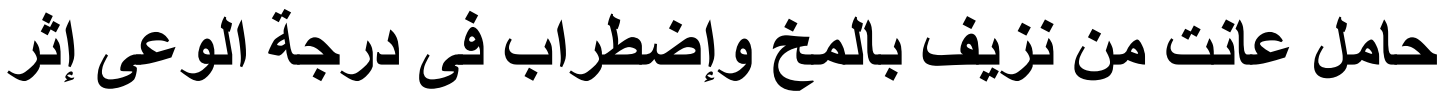 حادث سيارة (تقريز حالة)}

طه محمد رشاد عامر، يسمين حربى صادق، عبد الحميد عزت جابر مستثفى شرم الدولى، شرم الثيخ، مصر

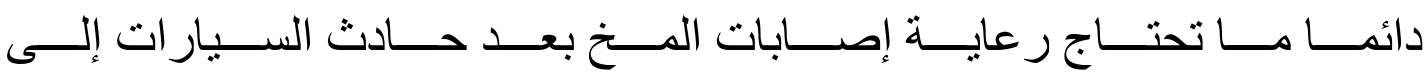

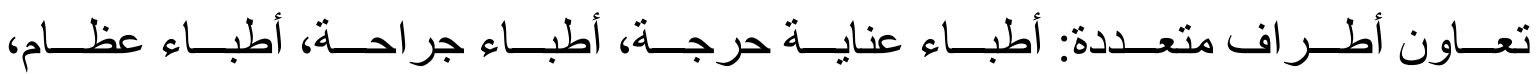

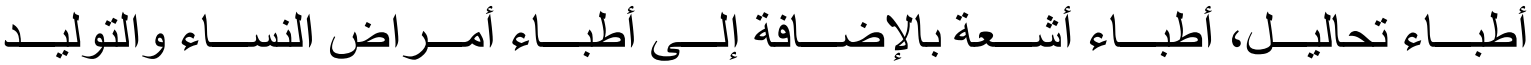

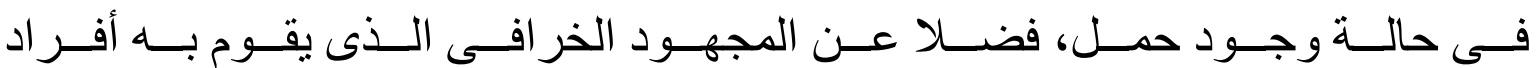

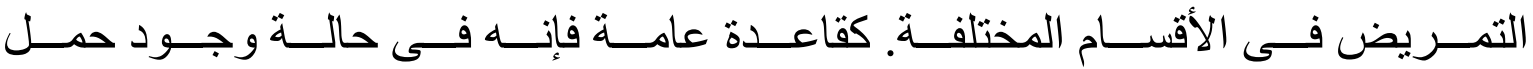

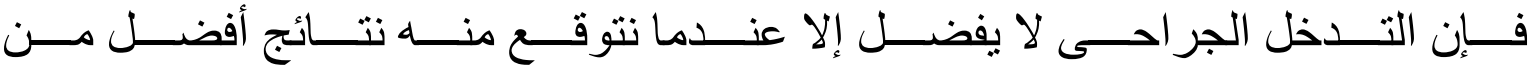

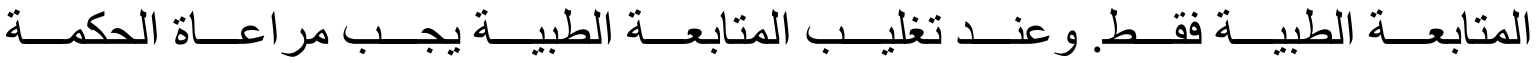

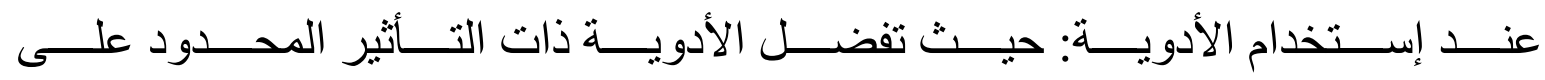
الجنين أو التى ليس لها خطورة عليه.

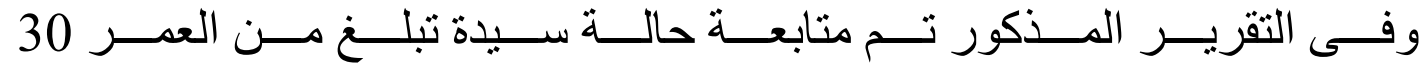

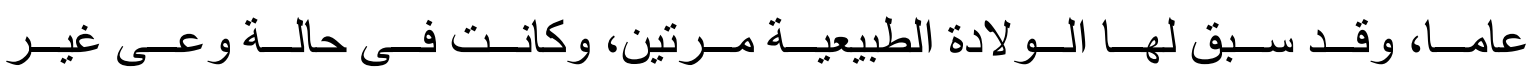

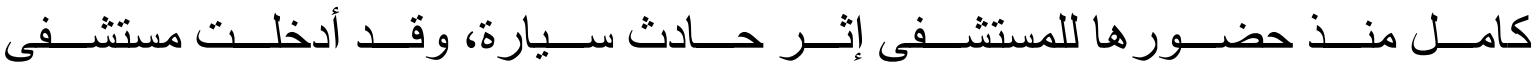

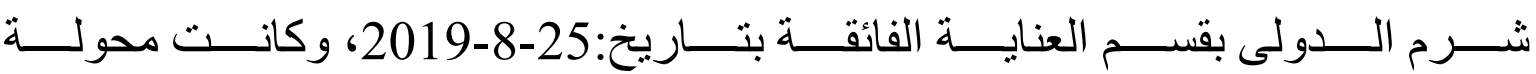

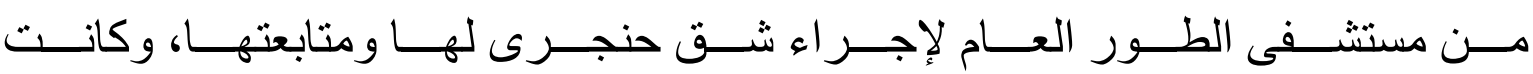

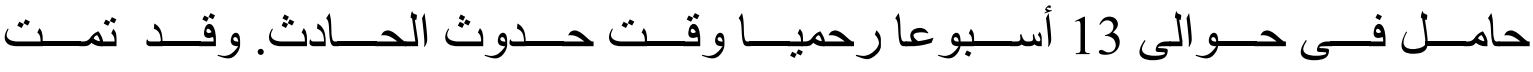

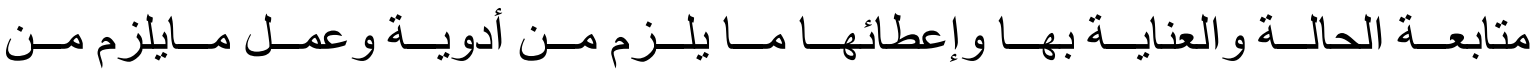

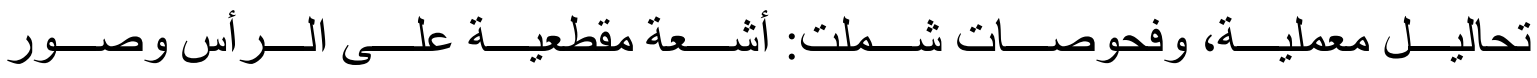

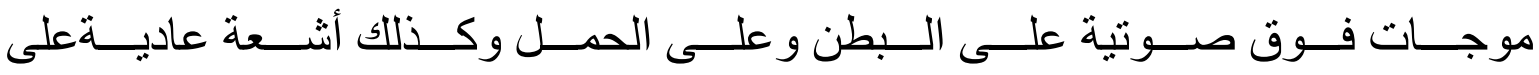




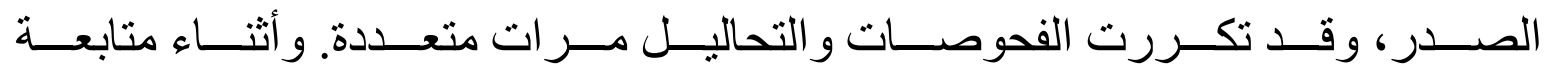

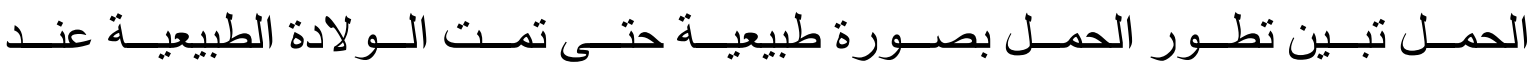

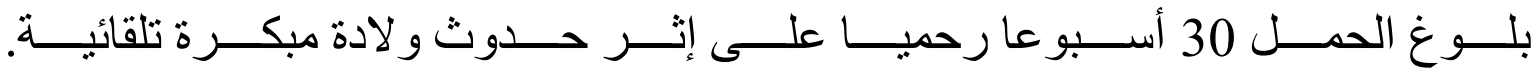

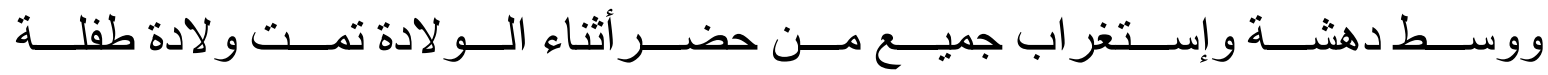

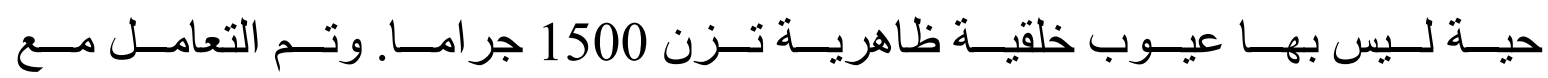

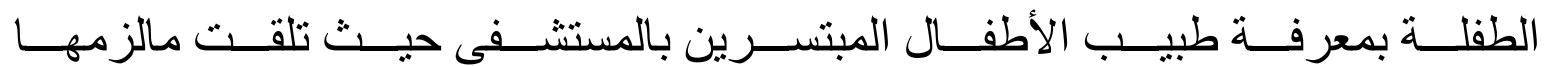

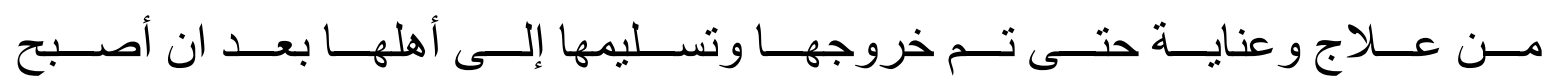

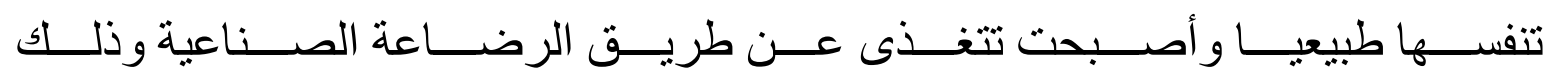
بعد ان مكثت فى قسم الأطفال المبتسرين لمدة 3 أسابيع. 